Original Research Paper

\title{
Reduction of Radiation Effects in Biomedical Imaging Detectors by Technology Change
}

\author{
${ }^{1}$ Essesse Songa Auguste, ${ }^{1}$ Wembe Tafo Evariste, ${ }^{2}$ Madinatou Salomon and ${ }^{3}$ Belinga Aboudou Jean \\ ${ }^{1}$ Department of Physics, Faculty of Science, University of Douala, B.O. Box, 24157 Douala, Cameroon \\ ${ }^{2}$ Department of Physics, Faculty of Science, University of Yaoundé I, B.O. Box 812 Yaoundé, Cameroon \\ ${ }^{3}$ Department Electrical Engineering, ENSET, University of Douala, B.O. Box, 24157 Douala, Cameroon
}

\author{
Article history \\ Received: 19-05-2021 \\ Revised: 09-06-2021 \\ Accepted: 10-06-2021 \\ Corresponding Author: \\ Wembe Tafo Evariste \\ Department of physics, Faculty \\ of science, University of \\ Douala, B.O. Box, 24157 \\ Douala, Cameroon \\ Email: twembee@yahoo.fr
}

\begin{abstract}
Silicon is a preferred material in the design of medical imaging detectors. It enables reliable and inexpensive detectors to be produced. However, the operation of this material is compromised when irradiated with high radiation of more than $5 \times 10^{14}$ particles $/ \mathrm{cm}^{2}$ of high energy neutrons. The defects caused transform the electrical properties. Therefore, this loss of information compromises the reconstruction of important events. This research paper aims to make a scientific contribution to the reduction of this radiation effects. The proposed solution is obtained by changing the silicon material generally used to design the detectors by a carbon nanotube material. The use of carbon nanotube material allows the detector to reduce the effects of radiation and leakage currents. A particular observation was made on the linear attenuation coefficient $\mu$, the radiation length $X_{0}$ and the width of the forbidden band $E_{g}$. Our results show the best characteristics for a carbon nanotube material compared to silicon. For a cross thermal section equal to $\sigma_{S i}=\sigma_{c}=2 \times 10^{-3} \mathrm{~b}$, the carbon linear attenuation coefficient is great her than the silicon $\left(\mu_{S i}=9.979 \times 10^{-5} \mathrm{~cm}^{-1}<\mu_{C}=3.533 \times 10^{-4} \mathrm{~cm}^{-1}\right)$, For a maximum effective cross section of equal diffusion $\sigma_{C}=1009 \times 10^{-3} \mathrm{~b},\left(\mu_{S i}=0.503 \times 10^{-3}\right.$ $\mathrm{cm}^{-1}<\mu_{C}=1.732 \times 10^{-3} \mathrm{~cm}^{-1}$ ), for a minimum effective cross section of equal diffusion $\sigma_{S i}=\sigma_{C}=10.09 \times 10^{-3} b, \mu_{S i}=0.503 \times 10^{-3} \mathrm{~cm}^{-1}<\mu_{C}=1.732 \times 10^{-3}$ $\mathrm{cm}^{-1} . X_{0_{s i}}=22.009 \mathrm{~g} / \mathrm{cm}^{2}$ and $X_{0_{c}}=42.969 \mathrm{~g} / \mathrm{cm}^{2}, E_{g_{s i}}=1.2 \mathrm{e} . \mathrm{V}$ and $E_{g_{s i}}=$ $5.5 \mathrm{e}$.V. From these results, the carbon material has an attenuation coefficient at least three times higher than that of the silicon material.
\end{abstract}

Keywords: Silicon, Carbon, Technology Change, Radiation, Biomedical Imaging, Attenuation Coefficient, Material

\section{Introduction}

With the progress of microelectronics, we are witnessing the improvement of the living conditions of human beings in several areas such as communication, transport, medicine (Lanzarotti, 1990; Bulletin, 1985; Lemoine, 2015) etc. This is why man is always in search of well-being in the face of the difficulties encountered. It is in the same order, for example, that Research programs in particle physics, nuclear physics, astrophysics and medicine are growing in number (Evariste et al., 2010). In biomedical imaging, most of the detectors used are made from silicon semiconductors on a microscopic scale (Davia, 2003). However, the radiations resulting from the collision between the electromagnetic radiation and the matter very often affect the detector by its effects of radiations (Wunstorf, 1997; Claeys and Simoen, 2002). This subsequently leads to the deterioration of the sensors, thus modifying the information of the expected results. However, by virtue of its properties, diamond being essentially made up of carbon, we find that the latter is an excellent resistant to radiation (Besson, 2015; Bortolamiol, 2015). For this purpose, we propose in this article, the change of the silicon material generally used by a carbon nanotube material. According to the research of Chantepie (2008; Besson, 2015), it emerges that a good detector is chosen according to the thickness of the substrate, the width of the forbidden band, the density of the material. Despite the wealth of recent research on the characteristics of a good detector, no paper has noted the change in technology from silicon material to carbon nanotube material. Therefore, our study focused on an 
analytical and numerical analysis of the characteristics of carbon nanotubes. This article is structured as follows, firstly, we will do an analytical and comparative study where we will compare the attenuation coefficient, the length of radiation and band gap width of silicon material relative to carbon nanotube material. Then the continuation, one will make a numerical study in which we seek the variations of the coefficients of these two materials according to the thermal effective section and maximum and minimum cross sections of diffusion. To better understand our work, the results obtained and the interpretation of these results will be the subject of our attention in turn. At the end, we will make a conclusion.

\section{Analytical and Comparative Study}

A study has shown that two effects of radiation damage detectors when exposed to electromagnetic radiation (Wunstorf, 1997; Claeys and Simoen, 2002). As we said above, this study is based on the analysis and comparison of three determining parameters for the choice of a good detector exposed to electromagnetic radiation. We will begin our study with the linear attenuation coefficient of a material given by formula (Chantepie, 2008):

$$
\mu=N_{A} \frac{\rho}{A} \sigma\left(\mathrm{cm}^{(-1)}\right)
$$

where, $\mu$ is the linear attenuation coefficient, $\rho$ is the density of the material, $N_{A}$ is the Avogadro constant and $\sigma$ is the cross section and $A$ is the molar mass of the material.

Then, the coefficients of silicon and carbon are respectively given by:

$$
\begin{aligned}
& \mu_{S i}=\rho_{S i} \frac{N_{A}}{A_{S i}} \sigma_{S i}\left(\mathrm{~cm}^{-1}\right) \\
& \mu_{c}=\rho_{c} \frac{N_{A}}{A_{c}} \sigma_{c}\left(\mathrm{~cm}^{-1}\right)
\end{aligned}
$$

By differentiating these coefficients, we obtain the following expression:

$$
\mu_{c}-\mu_{S i}=N_{A}\left(\sigma_{C} \frac{\rho_{c}}{A_{c}}-\sigma_{S i} \frac{\rho_{S i}}{A_{S i}}\right)
$$

and the simplest case, with equal section this expression becomes:

$$
\mu_{C}-\mu_{S i}=N_{A} \sigma\left(\frac{\rho_{c}}{A_{c}}-\frac{\rho_{S i}}{A_{S i}}\right)\left(c m^{-1}\right)
$$

where the values of the constants $\rho_{c}$ and $\rho_{S i}$ are taken from the Table 1 (Chantepie, 2008; Besson, 2015).
Another determining parameter for the choice of a good detector is the length of radiation noted $X_{0}$, which is actually a physical quantity of choice of a material relating to the loss of energy by electromagnetic interaction (Isabelle, 2009)

The expression for this length is given by (Eidelman, 2004):

$$
X_{0}=\frac{716.4 \times A}{Z(Z+1) \ln \frac{287}{\sqrt{z}}}\left(\mathrm{~g} / \mathrm{cm}^{2}\right)
$$

where, $A$ and $Z$ are the molar mass and atomic number of the material, respectively.

Then, the radiation lengths of silicon and carbon are respectively given by:

$$
\begin{aligned}
& X_{0_{S i}}=\frac{716.4 \times A_{S i}}{Z_{S i}\left(Z_{S i}+1\right) \ln \frac{287}{\sqrt{z_{S i}}}}\left(\mathrm{~g} / \mathrm{cm}^{2}\right) \\
& X_{0_{C}}=\frac{716.4 \times A_{C}}{Z_{C}\left(Z_{C}+1\right) \ln \frac{287}{\sqrt{z_{C}}}}\left(\mathrm{~g} / \mathrm{cm}^{2}\right)
\end{aligned}
$$

and by differentiating these two lengths $X_{0_{c}}-X_{0_{s i}}$, we get the expression:

$716,4\left(\frac{A_{C}}{z_{C}\left(z_{C}+1\right) \ln \frac{287}{\sqrt{z_{C}}}}-\frac{A_{S i}}{z_{S i}\left(z_{S i}+1\right) \ln \frac{287}{\sqrt{z_{S i}}}}\right)$

In addition, it should be noted that the choice of the material to be used will be that which has the greatest length of radiation. The choice of a detector also depends on the band gap $E_{g}$, the larger this width the considerably good and the leakage current considerably decreases (Chantepie, 2008). By observing the Table 1, we can easily note this width and make the difference between the width of the forbidden band of the material with the carbon nanotube and that of silicon.

\section{Digital Study}

For this study, we will approach three cases of figures. In the first, given that we are in the high frequencies, we will work with the thermal cross section, the order of magnitude of which varies between $2 \times 10^{-27} \mathrm{~cm}^{2}$ and 3.4 $\times 10^{-27} \mathrm{~cm}^{2}$. With equal cross section of carbon and silicon, we collected the data in Table 2. These data will allow us to simulate on MATLAB, the variations of the attenuation coefficients of carbon and silicon as a function of the cross section. The second and third 
numerical study relate respectively to the reading of the attenuation coefficients maximum and minimum cross sections of diffusion the data of which are recorded on Table 3 and 4 (Thiollière, 2005).

\section{Presentation of the Results}

Table1 (Chantepie, 2008; Besson, 2015) shows the characteristics of some semiconductors. These characteristics permitted us to determine the values of the densities and the widths of the forbidden bands of the materials.

With an equal cross section, the difference $\mu_{C^{-}} \mu_{S i}$ of Eq. (5) is equal to $2.5351 .10^{-1} \mathrm{~cm}^{-1}$. The ratio between the attenuation coefficients of carbon and silicon determined is $\frac{\mu_{C}}{\mu_{S i}} \cong 3.54$.

On the other hand, the radiation lengths of silicon and carbon are respectively:

$$
\left\{\begin{array}{l}
X_{0 S i}=22.009 \mathrm{~g} / \mathrm{cm}^{2} \\
X_{0 C}=42.969 \mathrm{~g} / \mathrm{cm}^{2}
\end{array}\right.
$$

The difference and the ratio between the two radiation lengths gives us respectively:

$$
\left\{\begin{array}{l}
X_{0 C}-X_{0 S i}=20.96 \mathrm{~g} / \mathrm{cm}^{2} \\
\text { and } \\
\frac{X_{O C}}{X_{0 S i}}=1.952337
\end{array}\right.
$$

Moreover, using Table 1 for the widths of the forbidden bands, we note that:

$$
\left\{\begin{aligned}
E_{g S i} & =1.2 e . V \\
& \text { and } \\
E_{g C} & =5.5 e . V
\end{aligned}\right.
$$

Table 1: Inventory of the main characteristics of materials used for particle detection

\begin{tabular}{lllllllll}
\hline $\begin{array}{l}\text { Material } \\
\text { Unit }\end{array}$ & $\mathrm{Z}$ & $\begin{array}{l}\text { Density } \\
\mathrm{g} / \mathrm{cm}^{-3}\end{array}$ & $\begin{array}{l}E_{g} \\
\mathrm{eV}\end{array}$ & $\begin{array}{l}E_{p} \\
\mathrm{eV}\end{array}$ & $\begin{array}{l}\mu_{n} \\
\mathrm{Cm}^{2} / \mathrm{Vs}\end{array}$ & $\begin{array}{l}\tau_{e}^{-} \\
\mathrm{s}\end{array}$ & $\begin{array}{l}\mu_{p} \\
\mathrm{~cm}^{2} /(\mathrm{Vs})\end{array}$ & $\begin{array}{l}\tau_{p}^{+} \\
s\end{array}$ \\
\hline $\mathrm{Si}$ & 14.0 & 2.32 & 1.12 & 3.60 & 1450 & $1.10^{-3}$ & 450 & $2.10^{-3}$ \\
$\mathrm{Ge}$ & 32.0 & 5.33 & 0.67 & 2.96 & 3900 & $1.10^{-3}$ & 1900 & $1.10^{-3}$ \\
$\mathrm{AsGa}$ & 32.0 & 5.32 & 1.43 & 4.30 & 8000 & $10.10^{-9}$ & 400 & $100.10^{-7}$ \\
$\mathrm{CdTe}$ & 50.0 & 5.85 & 1.52 & 4.43 & 1100 & $3.10^{-6}$ & 100 & $2.10^{-6}$ \\
$\mathrm{Cd}_{0,9} \mathrm{Zn}_{0,1} \mathrm{Te}$ & 49.1 & 5.78 & 1.57 & 4.64 & 1000 & $3.10^{-6}$ & 80 & $1.10^{-6}$ \\
diamond & 6.0 & 3.52 & 5.50 & 13.00 & 1800 & $10^{-3}$ & 1200 & $<10^{-3}$ \\
$\mathrm{a}-\mathrm{Si}$ & 14.0 & 2.30 & 1.80 & 4.00 & 1 & $7.10^{-9}$ & $5.10^{-3}$ & $4.10^{-6}$ \\
$\mathrm{a}-\mathrm{Se}$ & 34.0 & 4.30 & 2.20 & 7.00 & $5.10^{-3}$ & $1.10^{-6}$ & $1.410^{-1}$ & $1.10^{-6}$ \\
$\mathrm{HgI}_{2}$ & 62.0 & 6.40 & 2.13 & 4.20 & 100 & $1.10^{-6}$ & 4 & 2 \\
$\mathrm{PbI}_{2}$ & 62.7 & 6.20 & 2.32 & 4.90 & 8 & & 2 & $1.10^{-5}$ \\
\hline
\end{tabular}

Table 2: Statement of the attenuation coefficients of silicon and carbon materials as a function of the cross section

\begin{tabular}{lcccccccl}
\hline$\sigma\left(\mathrm{cm}^{2}\right) \times 10^{-27}$ & 2 & 2.1 & 2.2 & 2.3 & 2.4 & 2.5 & 2.6 & \\
$\mu_{C}\left(\mathrm{~cm}^{-1}\right) \times 10^{-6}$ & 353.3 & 370.965 & 388.63 & 406.295 & 423.96 & 441.625 & 459.29 & \\
$\mu_{S i}\left(\mathrm{~cm}^{-1}\right) \times 10^{-6}$ & 99.796 & 104.7858 & 109.7756 & 114.7654 & 119.7552 & 124.745 & 129.7348 & \\
$\sigma\left(\mathrm{cm}^{2}\right) \times 10^{-27}$ & 2.7 & 2.8 & 2.9 & 3 & 3.1 & 3.2 & 3.33 .4 & \\
$\mu_{C}\left(\mathrm{~cm}^{-1}\right) \times 10^{-6}$ & 476.955 & 494.62 & 512.285 & 529.95 & 547.615 & 565.28 & 582.945 & 600.61 \\
$\mu_{S i}\left(\mathrm{~cm}^{-1}\right) \times 10^{-6}$ & 134.7246 & 139.7144 & 144.7042 & 149.694 & 154.6838 & 159.6736 & 164.6634 & 169.6532 \\
\hline
\end{tabular}

Table 3: Statement of the attenuation coefficients of silicon and carbon materials as a function of the maximum cross section of diffusion

\begin{tabular}{llllllllll}
\hline$\sigma\left(\mathrm{cm}^{2}\right) \times 10^{-25}$ & 9.830 & 9.790 & 9.74 & 9.86 & 10.09 & 9.810 & 9.780 & 9.840 & 10.08 \\
$\mu_{C}\left(\mathrm{~cm}^{-1}\right) \times 10^{-3}$ & 1.735 & 1.728 & 1.719 & 1.741 & 1.781 & 1.732 & 1.727 & 1.737 & 1.779 \\
$\mu_{S i}\left(\mathrm{~cm}^{-1}\right) \times 10^{-3}$ & 0.490 & 0.488 & 0.485 & 0.491 & 0.503 & 0.489 & 0.487 & 0.490 & 0.502 \\
\hline
\end{tabular}

Table 4: statement of the attenuation coefficients of silicon and carbon materials as a function of the minimum cross section of diffusion

\begin{tabular}{llllllllll}
\hline$\sigma\left(\mathrm{cm}^{2}\right) \times 10^{-25}$ & 9.230 & 9.390 & 9.340 & 9.220 & 9.650 & 9.250 & 9.740 & 9.240 & 9.360 \\
$\mu_{C}\left(\mathrm{~cm}^{-1}\right) \times 10^{-3}$ & 1.629 & 1.658 & 1.649 & 1.628 & 1.704 & 1.633 & 1.659 & 1.631 & 1.705 \\
$\mu_{S i}\left(\mathrm{~cm}^{-1}\right) \times 10^{-3}$ & 0.460 & 0.468 & 0.465 & 0.459 & 0.481 & 0.461 & 0.468 & 0.460 & 0.481 \\
\hline
\end{tabular}


The difference and the ration between the forbidden bandwidths give us respectively:

$$
\left\{\begin{array}{c}
E_{g C}-E_{g S i}=4.3 e . V . \\
\text { and } \\
\frac{E_{g C}}{E_{g S i}}=4.9107
\end{array}\right.
$$

For the numerical study, with an equal thermal cross section and variable between $2 \times 10^{-27} \mathrm{~cm}^{2}$ and $3.4 \times 10^{-27}$ and. We have recorded the values of attenuation coefficients of silicon and carbon materials as a function of the cross section in Table 2 (Bauer et al., 1998).

By also making the ratio of the means between the carbon and silicon attenuation coefficient for Table 2, 3 and 4, we find respectively:

$$
\frac{\mu_{C 2}}{\mu_{S i 2}}=3.540, \frac{\mu_{C 3}}{\mu_{S i 3}}=3.548 \text { and } \frac{\mu_{C 4}}{\mu_{S i 4}}=3.544
$$

\section{Interpretation of Results}

The analytical study allowed us notice that:

$$
\left\{\begin{array} { l } 
{ \mu _ { C } - \mu _ { S i } > 0 } \\
{ X _ { 0 C } - X _ { 0 S i } > 0 \text { and } } \\
{ E _ { g C } - E _ { g S i } > 0 }
\end{array} \left\{\begin{array}{l}
\frac{\mu_{C}}{\mu_{S i}}>1 \\
\frac{X_{0 C}}{X_{0 S i}}>1 \\
\frac{E_{g C}}{E_{g S i}}>1
\end{array}\right.\right.
$$

According to these results for this study, we find that the carbon nanotube material has a better attenuation coefficient $\left(\mu_{C}-\mu_{S i}>0\right.$ and $\left.\frac{\mu_{C}}{\mu_{S i}}>1\right)$, has a greater radiation length and a greater band gap compared to the silicon material. We can therefore say that the carbon material biomedical imaging detector will better reduce the effects of radiation and leakage currents compared to silicon material.

Numerically, the Fig. 1, 2 and 3 below, showing the curves of the attenuation coefficients of carbon nanotube materials and silicon. We notice that in these three cases, the curves of the attenuation coefficients of carbon radiation are always above those of silicon whatever the nature of the nature of the effective section. On the other hand, we find that the attenuation coefficient of the carbon nanotube material increases with the cross section for Fig. 1 but, with a few exceptions, can even reach a peak following a certain value of the effective section. At the same time for Fig. 1, the attenuation coefficient of silicon material first decreases and then increases slightly with the cross section. For Fig. 2 and 3, the variation in the attenuation coefficient of the carbon and silicon material varies very little as a function of the effective section. All these remarks lead us to say that with a few exceptions, the attenuation coefficient of a material increases the effective section of the latter. Therefore, the greater the cross section of the material, the more the detector can considerably reduce the effects of radiation. The maximum value of the radiation attenuation coefficient of the carbon material is reached when the effective section of the latter takes the value $28 \times 10^{-27} \mathrm{~cm}^{2}$.

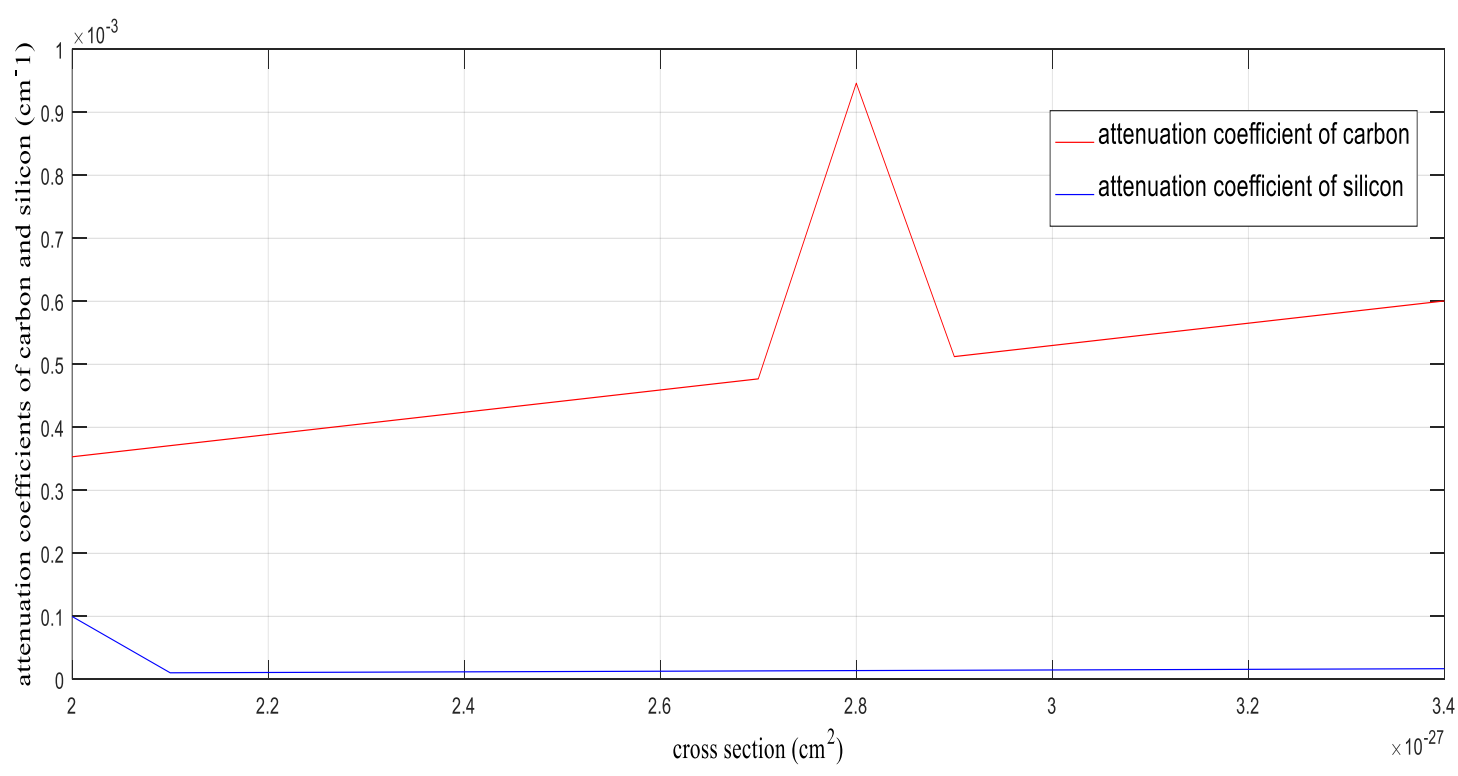

Fig. 1: Curves of the attenuation coefficients of carbon and silicon as a function of the thermal cross section 


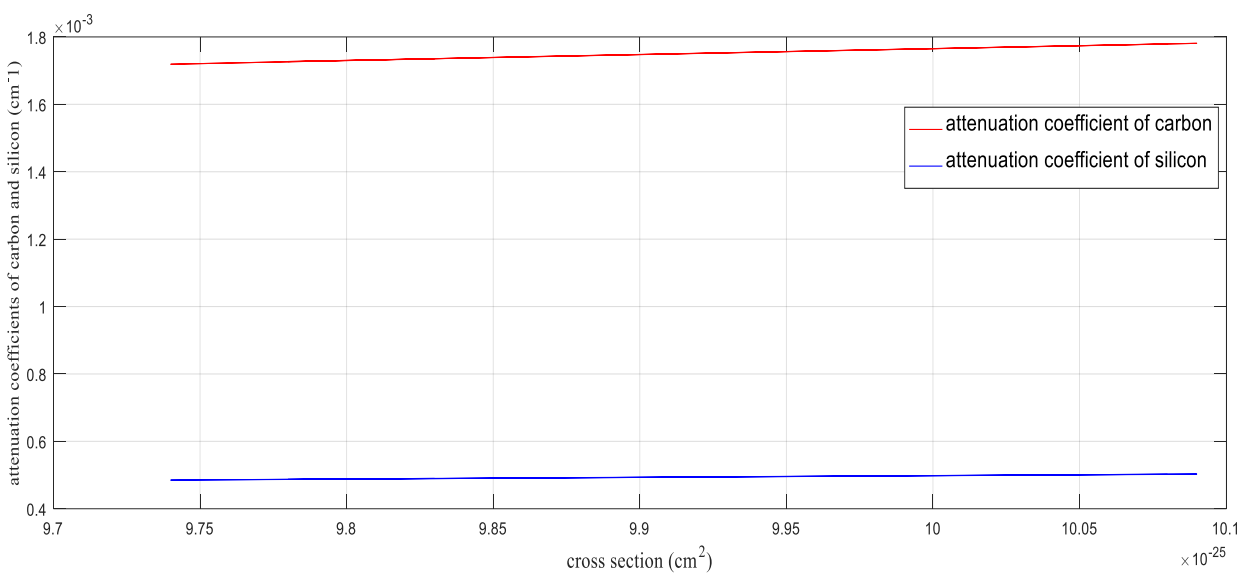

Fig. 2: Curves of the variations of the attenuation coefficient of silicon and carbon materials as a function of the maximum cross section of diffusion

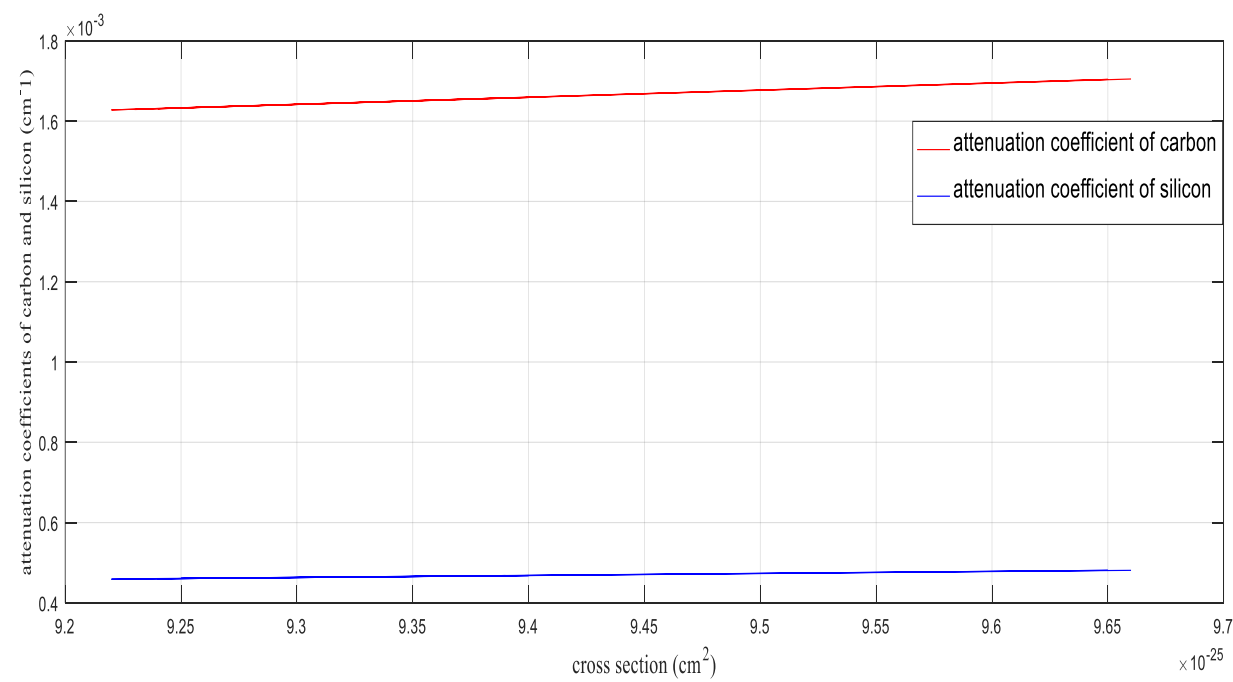

Fig. 3: Curves of the variations of the attenuation coefficient of silicon and carbon materials as a function of the minimum cross section of diffusion

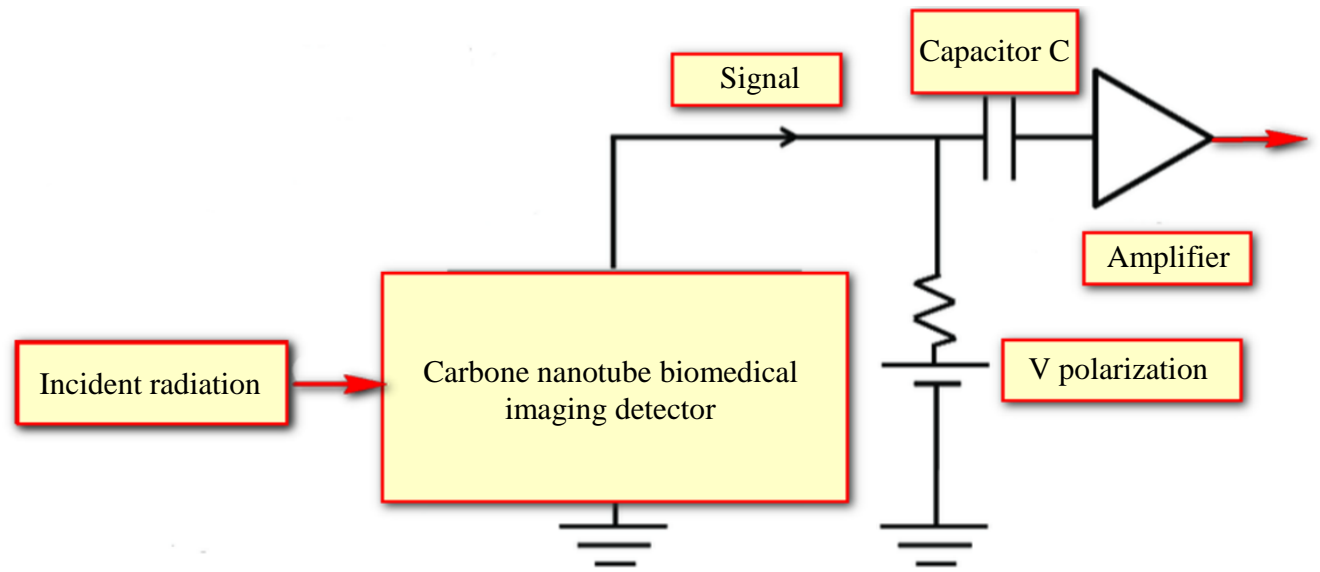

Fig. 4: diagram of the biomedical imaging detection device 


\section{Presentation of the Carbon Nanotube Biomedical Imaging Detector}

In a simplified way, here is the functional diagram of the detection device (Legou, 2002) this diagram is a representation of the general operating principle of the detection chain by semiconductor detectors in imaging, see Fig. 4. This principle can be summed up in four steps. The first step is materialized by the interaction of incident radiation in the active material of the detector. This interaction is accompanied by a partial deposition of the energy of the incident radiation on the one hand, but also by the creation of charge carriers (electron/ion pairs) on the other hand. The second stage is marked by the transport, the collection of a signal and the measurement of an induced current linked to the movement of these charge carriers created in the first stage (Knoll and Wiley, 1989). The third step is marked by the processing and amplification of the signal delivered by the detector by an electronic circuit (Lemoine, 2015). In other words, this part is reserved for the reduction of the disturbances which accompany the signal to a certain extent but also the amplification of the latter (Foulquier and Radiol, 2010). The last step marked by the transfer of the signal to a data acquisition and storage system for use.

\section{Conclusion}

At the end of our research, the aim of which was to propose one of the solutions to reduce the effects of radiation on the biomedical imaging detector. We recall that the man goal is to reduce the effects of radiation on the detection device. On the other hand, it was also a question of reducing the leakage currents. The results obtained show that the ratio between the attenuation coefficients of carbon and silicon $\left(\frac{\mu_{C}}{\mu_{S i}}\right)$ is approximately equal to 3.5. In the same perspective, the ration between the forbidden bandwidths $\left(\frac{E_{g C}}{E_{g s i}}\right)$ give us 4.9107. In other words, the car material at least three and a half times more resistant to radiation than the silicon material on the one hand. On the other hand, the carbon material can significantly reduce the leakage currents five times compared to the silicon material. In view of all observations, we can say thatcarbon nanotubes are more resistant to the effects of radiation compared to silicon material on the one hand. On the other hand, because of the large value of band gap, carbon nanotubes reduce leakage currents that can affect the system. It is therefore possible to reduce not only the effects of radiation on the imaging detector, but also to considerably reduce the leakage currents which can disturb the information expected when using the carbon material. We can say that carbon nanotube is near future and reliable solutions for biomedical imaging. For the moment, the limits of our work lie on experimentation. This is why, in a near future, we would like to work on themes such as: Experimental study of data from carbon and silicon detector intended for medical imaging. General study of materials to determine the best medical imaging detector et where possible, design the best imaging detector.

\section{Author's Contributions}

Essesse Songa Auguste: Performed some of the experiments through simulation software.

Wembe Tafo Evariste: Project leader, data interpretation and contribute to writing of the peper.

Madinatou Salomon and Belinga Aboudou Jean: Contribute to the writing of the paper.

\section{Ethics}

The corresponding author confirms that all of the other authors have read and approved the manuscript and no ethical issues involved.

\section{References}

Bauer, R. W. Anderson, J. D., Grimes, S. M., Knapp, D. A., \& Madsen, V. A. (1998). Application of a simple Ramsauer model for neutron total cross sections. Nuclear science and engineering, 130(3), 348-360. https://www.tandfonline.com/doi/abs/10.13182/NSE 98-A2011

Besson, A. (2015). Détecteurs à semi-conducteur conducteurs, juin 2015.

Bortolamiol, T. (2015). Nanotubes de carbone biparois: fonctionnalisation et détection in vitro (Doctoral dissertation). https://oatao.univ-toulouse.fr/13943/

Bulletin, A. (1985). Microelectronics based automation technologies and development, ${ }^{\circ} 2$ Novembre 1985, Centre for science and technology development, united Nations New-york

Chantepie, B. (2008). Study and realization of fast low noise electronics for a hybrid pixel X-ray detector intended for small animal imaging (Doctoral dissertation, Université de la Méditerranée-Aix-Marseille II). https://tel.archives-ouvertes.fr/tel-00366861/

Claeys, C., \& Simoen, E. (2002). Radiation effects in advanced semiconductor materials and devices (Vol. 57). Springer Science \& Business Media. ISBN-10: 3540433937.

Davia, C. (2003). Détecteurs au silicium radiorésistants: une solution d'avenir » université Brunei, RoyaumeUni, 2003. 
Eidelman, S. (2004). Review of particle physics. Particle Data Group. 2004.

Evariste, W. T., Hong, S., Q., Jie, K., \& Tongxi, W. A. N. G. (2010). Design and simulation of Gaussian shaping amplifier made only with CMOS FET for FEE of particle detector, Nuclear Science and Techniques Vol.21, No 5, October 2010 P.312-315

Foulquier, J. N. and Radiol, J. (2010). Technical parameters to decrease the radiation dose from conventional and digital radiograhs. 2010.

Knoll, G. F., \& Wiley, E. D. (1989). Radiation detection a mea surement., 1989. ISBN-10:0470131489

Lanzarotti, M. (1990). Micro-electronic automation: challenges for the Third World. Third World Review, 285-309. https://www.jstor.org/stable/23591503

Legou, T. (2002). Etude et réalisation d'une chaîne d' instrumentation numérique rapide pour $l^{\prime}$ identification des ions (Doctoral dissertation, Université de Caen). https://tel.archivesouvertes.fr/tel-00002517/
Lemoine, T. (2015). Imagerie médicale par rayons XCaractérisation des détecteurs de rayons $\mathrm{X}$. https://www.techniques-ingenieur.fr/basedocumentaire/biomedical-pharma-th15/imageriemedicale-42607210/imagerie-medicale-par-rayons$\mathrm{x}$-med202/

Thiollière, N. (2005). Mesure des sections efficaces de diffusion élastique des neutrons sur le carbone et le fluor dans le domaine épithermique sur la plate-forme PEREN (Doctoral dissertation, Université JosephFourier-Grenoble I). https://tel.archivesouvertes.fr/tel-00011285/

Isabelle, W. (2009). Les Detecteurs en physique des particules, 2009.

Wunstorf, R. (1997). Radiation hardness of silicon detectors: current status. IEEE Transactions on Nuclear Science, 44(3), 806-814. https://ieeexplore.ieee.org/abstract/document/603757/ 